\title{
HIGH RESOLUTION X-RAY DIFFRACTION INVESTIGATIONS OF Si/SiGe QUANTUM WELL STRUCTURES AND Si/Ge SHORT-PERIOD SUPERLATTICES
}

\author{
G. Bauer, E. Koppensteiner, P. IInMberger
}

Institut für Halbleiterphysik, Johannes Kepler Universität Jinz, 4040 Linz, Austria

$$
\text { J. Nützel, G. Abstreiter }
$$

Walter Schottky Institut, TU München, D-8046 Garching, Germany

\author{
H. Kibbel, H. Presting and E. Kasper \\ Daimler Benz AG, Forschungszentrum \\ Wilhelm Runge Straße 11, D-7900 Ulm, Germany
}

\begin{abstract}
Double crystal and triple axis X-ray diffractometry was used to charactcrize the structural properties of $\mathrm{Si} / \mathrm{Si}_{1-x} \mathrm{Ge}_{x}$ multiquantum well samples grown pseudomorphically on Si(001) substrates, as wcll as of short-period $\mathrm{Si}_{9} \mathrm{Ge}_{6}$ superlattices grown by molecular beam epitaxy on rather thick stcp-graded $\mathrm{Si}_{1-x} \mathrm{Ge}_{x}(0<x<0.4,650 \mathrm{~nm}$ thick $)$ buffers followed by $550 \mathrm{~nm}$ $\mathrm{Si}_{0.6} \mathrm{Ge}_{0.4}$ layers. Reciprocal space maps around the (004) and (224) reciprocal lattice points yield direct information on the strain status of the layers in the heterostructure systems and in particular on the amount of strain relaxation.
\end{abstract}

PACS numbers: $68.65 .+\mathrm{g}, 61.10 .-\mathrm{i}, 68.55 . \mathrm{Jk}$

\section{Introduction}

Within the last two years considerable progress has been achieved in the growth of $\mathrm{Si} / \mathrm{SiGe}$ heterostructures and $\mathrm{Si}_{m} / \mathrm{Ge}_{n}$ short-period superlattices (SL's, $m$ and $n$ denote the numbers of $\mathrm{Si}$ and Ge monolayers) [1-4]. These advances have opened the prospect to fabricate electronic [1] and optoclectronic heterostructure devices on Si substrates [4] using at least partially the available advanced $\mathrm{Si}$-technology. In fact, various properties of $\mathrm{Si} / \mathrm{SiGe}$ heterostructures are nowadays becoming comparable to those of high quality $\mathrm{GaAs} / \mathrm{Ga}_{1-x} \mathrm{Al}_{x} \mathrm{As}$ heterostructures. 
Like in any other heterostructure system, the electronic properties of Si/SiGe heterostructures are governed by the conduction and valence band discontinuities at their interfaces. In contrast to GaAs/AlAs heterostructures for which the lattice mismatch is almost negligible, the mismatch between $\mathrm{Si}$ and $\mathrm{Ge}$ is so large (4.17\%) that the built-in strain alters drastically the band structure of the layers, as well as their band line up. The biaxial compressive or tensile strain in the layers causes shifts and splittings of the valence and conduction band edges. However, in these strained layer systems the selection of proper amount of strain actually offers a new degree of freedom for choosing a certain band ordering [1, 2]. For Si and $\mathrm{Si}_{1-x} \mathrm{Ge}_{x}$ alloys $(x<0.85)$ the six conduction band minima are at the $\Delta$-points along the $\langle 001\rangle$ directions of the Brillouin zone (close to the $X$-points). For a [001] growth direction, a pseudomorphic SiGe layer deposited on a Si substrate experiences a biaxial compressive strain, which has the consequence that the four in-plane conduction band minima $(\Delta 4)$ are lowered in energy with respect to the remaining two valleys $(\Delta 2)$ oriented with their main axis along growth direction. In the pseudomorphic SiGe layer the conduction band offset between the Si minima and the $\Delta 4$ minima of SiGe is comparatively small, whereas the $\Delta 2$ minima of $\mathrm{SiGe}$ are shifted upwards in energy. In this situation the difference in energy gaps is approximately equal to the valence band offset. This is a favourable condition for confining holes in SiGe wells.

For two-dimensional confinement of electrons a different concept is used: a relaxed SiGe buffer is grown on the [001] Si substrate. If a Si layer is deposited on this SiGe buffer, it experiences a tensile biaxial strain. Consequently, the $\Delta 2$ conduction band minima in the Si layer are lowered, whereas the $\Delta 4$ minima are raised in energy $[1,2,3]$. Then the $\Delta 2$ valleys in the strained Si layers define the conduction band minimum in the heterostructure. The top of the valence band under both conditions is always located in the material with the smaller gap, i.e. in the SiGe layers. The band line ups for these two cases are shown schematically in Fig. 1. Basing on these concepts both $n$-type modulation doping (for electrons in $\mathrm{Si}$ wells) and $p$-type modulation doping (for holes in SiGe wells) has been successfully demonstrated [2].

An important consequence of the lattice mismatch is the fact that the thickness of the strained layers is limited by a critical thickness. Beyond this thickness pseudomorphic growth (i.e. in-plane lattice constants between the subsequent layers of different chemical composition are equal) is no longer possible and strain relaxation occurs, usually by the introduction of misfit dislocations. The critical thickness varies from about $1000 \AA$ for $1 \%$ lattice mismatch to about $100 \AA$ for $2 \%$ lattice mismatch.

Recently substantial progress has been reported in the growth of $\mathrm{Si}_{n} \mathrm{Ge}_{m}$ short-period strained layer superlattices [4]. This progress was achieved by the growth of $\mathrm{Si} / \mathrm{Ge}$ structures on comparatively thick (about $1 \mu \mathrm{m}$ ) linearly graded [5] or step-graded [6] SiGe alloy buffers instead of single step buffers with constant Ge content. Whereas for thin $(200 \AA)$ single step buffers the dislocation density in the $\mathrm{SiGe} / \mathrm{Si}$ heterostructures or superlattices is as high as $10^{10} \mathrm{~cm}^{-2}$, a significant reduction of the number of threading dislocations by several orders of magnitude has been shown to occur [5-7], if the refined buffer concept is used. Indirectly, the 

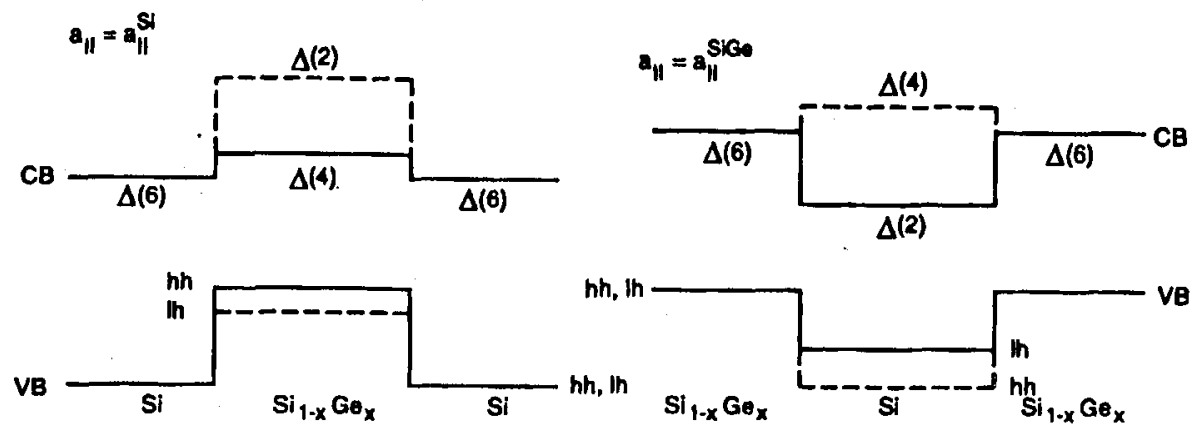

Fig. 1. Schematic diagram of the band alignment of $\mathrm{Si} / \mathrm{SiGe}$ heterostructures for in-plane lattice constants equal to that of silicon (left hand side) or to an SiGe alloy (right hand side). The splitting of the conduction band ( $\Delta$-minima), as well as of the light and heavy hole states is indicated.

superior structural quality of SiGe/Si heterostructure samples grown on graded SiGe buffer layers was demonstrated through the achievement of electron mobilities close to $200.000 \mathrm{~cm}^{2} /(\mathrm{V} \mathrm{s})$ at $T=4.2 \mathrm{~K}[8,9]$. Short-period strain-symmetrized $\mathrm{Si}_{m} \mathrm{Ge}_{n}$ superlattices have recently found considerable attention too, because of their sharp and intense luminescence in the near infrared [4, 10]. Quite recently even the observation of electroluminescence at room temperature has been reported [11] in strain adjusted $\mathrm{Si}_{6} \mathrm{Ge}_{4}$ superlattices.

The examples given above show the importance of the adequate control of the strain status in the active layers. For the given conduction and valence band deformation potentials [3], the strain tensor components determine the absolute value and sign of the energy shifts of the band extrema.

A nondestructive method for the determination of the strain status in strained layer systems is the use of high resolution $\mathrm{X}$-ray diffraction, which has been widely employed in the analysis of heterostructures and superlattices. Several investigations of the lateral strain $[12,13]$, as well as of the full-width at half maximum (FWHM) of X-ray diffractograms of SiGe buffer layers and superlattices (SL's) were reported so far using double crystal X-ray diffraction (DCD) $[14,15]$. Recently, for the analysis of complex multilayer structures the use of triple axis diffractometry (TAD) has been introduced, which allows the determination of the two-dimensional intensity distribution around reciprocal lattice points (RELP's) [16]. 


\section{Experimental}

It is the purpose of this paper to use DCD and TAD reciprocal space mapping of both symmetric (004) and asymmetric (224) Bragg diffraction peaks to compare the status of strain relaxation of an $\mathrm{Si} / \mathrm{SiGe}$ multiquantum well (MQW) structure grown on a Si substrate and three short-period $\mathrm{Si}_{m} \mathrm{Ge}_{n}(m=9, n=6)$ structures grown on thick step-graded SiGe buffers. The structural characteristics of substrate, buffer, and MQW or SL influence the positions of RELP's and the shape of iso-intensity contours around them. We show that these two-dimensional reciprocal lattice maps yield a wealth of structural information, usually much more than the conventional rocking curves [16-18].

A Bartels-type 4-crystal monochromator in the $\mathrm{Ge}(220)$ reflection mode was used in the primary beam, whereas in the diffracted beam either a slit with an opening angle of $0.05^{\circ}$ (DCD optics) or a two reflection $\mathrm{Ge}(220)$ channel-cut analyser crystal (TAD optics, detector opening angle 12 arcsec) was employed [16]. For all investigations $\mathrm{Cu} K_{\alpha_{1}}$ radiation was used with a wavelength $\lambda=$ $1.54059 \AA$. Besides the one-dimensional $\omega / 2 \Theta$ scans which scan the reciprocal space along a radial straight line from the (000) origin to the (004) or (224) RELP's, two-dimensional scans were performed in the plane of diffraction using triple axis optics by measuring a set of about hundred $\omega / 2 \Theta$ scans at different $\omega$ offset angles. The $\omega$-scan direction is along a circle around the $(000)$ center in reciprocal space. The step size was the same in both $\omega$ and $\omega / 2 \Theta$ scan directions. This reciprocal space mapping makes the shape of RELP's in two orthonormal directions in reciprocal space accessible and is extremely useful for the characterization of structural imperfections like strain gradients, interface roughness, bending or mosaicity [16-18]. All these imperfections give rise to diffuse scattering in the vicinity of the intensity maxima close to a RELP [19].

\subsection{Si/SiGe MQW structures}

Recently $p$-type modulation doped $\mathrm{Si} / \mathrm{Si}_{1-x} \mathrm{Ge}_{x} \mathrm{MQW}$ structures were grown by molecular beam epitaxy (MBE) on (001) Si substrates $(1500 \Omega \mathrm{cm}$ ) followed by an undoped Si epitaxial layer and subsequently the MQW structure doped properly for the observation of intersubband transitions between heavy hole states in the $\mathrm{Si}_{1-x} \mathrm{Ge}_{x}$ wells [20]. The MQW sample, on which X-ray data were taken, consisted of 10 repeats of nominally $50 \AA$ thick $\mathrm{Si}_{0.73} \mathrm{Ge}_{0.27}$ wells and $160 \AA$ thick Si barriers in which the central $60 \AA$ part was doped with boron $\left(2 \times 10^{18} \mathrm{~cm}^{-3}\right)$. A Si cap layer of $440 \AA$ was deposited on top.

In Fig. 2 in the insert a DCD rocking curve of the (004) Bragg reflection is shown. Apart from the substrate peak several superlattice peaks are identified from which the SL period can be determined readily. This sample shows subsidiary intensity maxima in between the main SL maxima which is a general diffraction phenomenon. Since the number of periods $N=10$, the number of subsidiary maxima has to be $N-2$, which is in this case 8 . The subsidiary maxima can be identified not only in between SLO and SL+1, but also in between the SLO and SL-1, SL-1 and SL-2 and even in between SL-2 and SL-3 peaks. The 


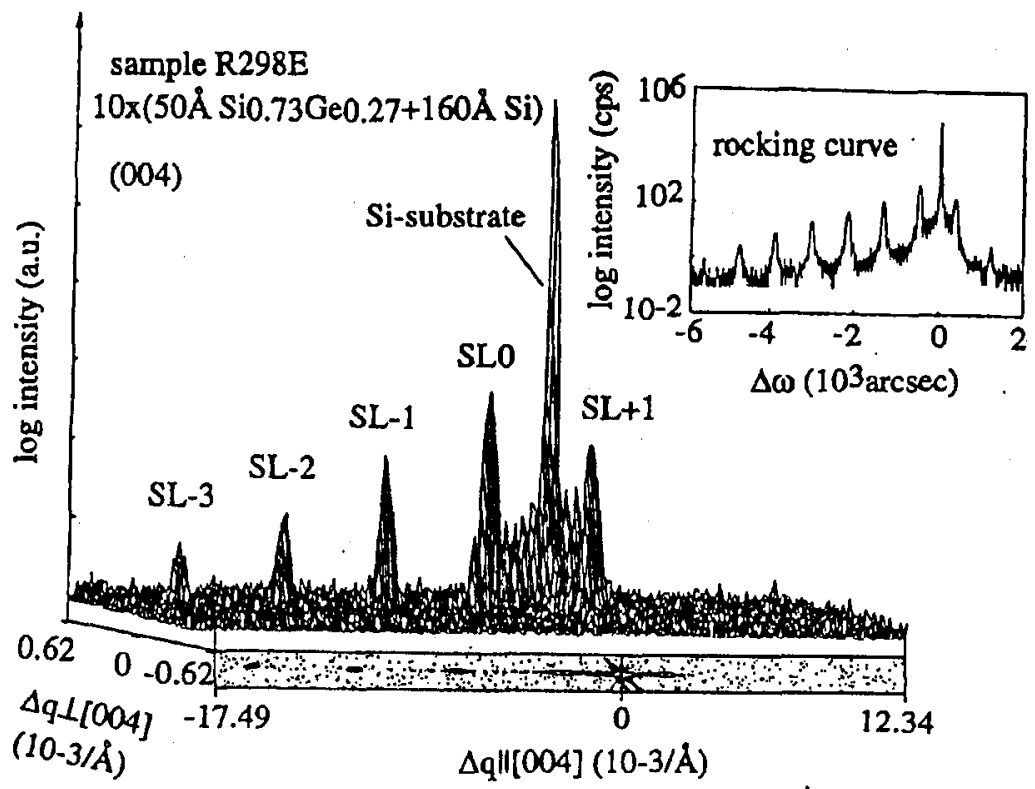

Fig. 2. Reciprocal space map of a pseudomorphic Si/SiGe MQW structure on Si(001) substrate in the vicinity of the (004) Bragg diffraction in the directions $q \|$ [004] direction and perpendicular to it. Contours of constant intensity (in a logarithmic scale) are shown around the Si substrate RELP and the superlattice SL-3 to SL+1 RELP's. Furthermore, a three-dimensional representation (intensity vs. the two $q$-directions) of this reciprocal space map is given as well. The insert shows the DCD rocking curve for the (004) Bragg diffraction with $\mathrm{Si}$ substrate peak and SL peaks from SL-6 to SL+1.

angular distance between SL peaks determines the SL period, whereas the total SL thickness is determined from the angular distance of two subsequent subsidiary maxima, which can be used to control the SL period calculated from the satellite spacings. From a fit to the DCD data it turns out that for this particular sample the actual parameters are - SL period: $227 \AA, d_{\mathrm{SiGe}}=52 \AA, d_{\mathrm{Si}}=175 \AA, \mathrm{Ge}$ content $x=0.23$.

In Fig. 2 also a reciprocal space map around the (004) Bragg reflection for the substrate and the SL+1, SL 0, SL $-1,-2,-3$ peaks are shown, which demonstrates the different FWHM's of the contours of equal intensity along [004] direction and perpendicular to it (i.e. [010] direction). The intensity in the reciprocal space map is also shown in a three-dimensional representation. In Fig. 3 the central part of Fig. 2 is enlarged for clarity for the demonstration of the subsidiary intensity maxima in between the SL0 and SL+1 superlattice (004) reflections for the same sample. The FWHM of the SL extrema along growth direction is 78 arcsec, which is due to the finite thickness of the entire MQW stack. However, perpendicular to growth direction the FWIIM is only 13 arcsec for the zero-order SL RELP (SL0), which indicates the extremely high crystalline perfection and the absence of any 


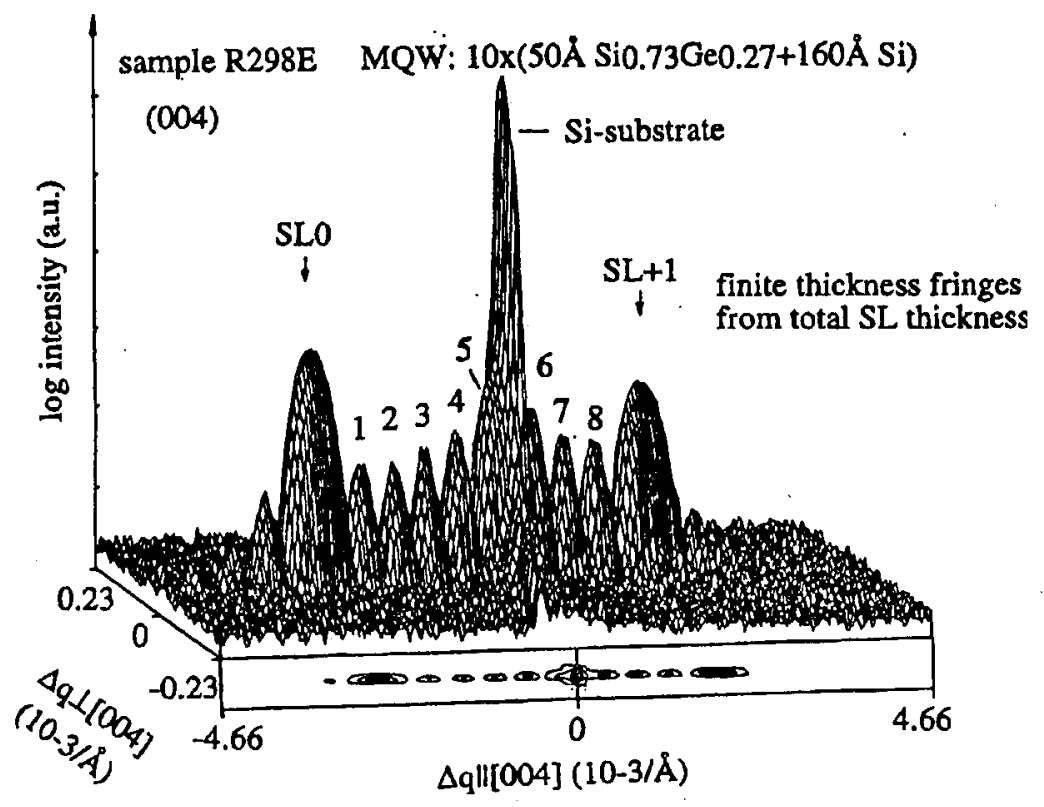

Fig. 3. Detail of Fig. 2 showing in between the SLO and SL+1 RELP clearly resolved finite thickness fringes $(N-2=8, N-$ number of QW's (10) in the MQW structure), indicating high structural perfection.

appreciable mosaicity. This fact is not astonishing, since the analysis of the (004) and the (224) reciprocal space maps yields that the mean in-plane lattice constant of the MQW constituent layers is exactly equal to that of the unstrained Si lattice constant. In the two-dimensional maps, the peak positions are characterized by the two orthogonal coordinates $q_{[004]}$ and $q_{[220]}$ (where $q$ denotes the wave vector in reciprocal space) which are related to the mean in-plane lattice constant $a_{p, \mathrm{SL}}$ and the one in growth direction $\left(a_{n, \mathrm{SL}}\right)$ by

$$
q_{[004]}=4 / a_{n, \mathrm{SL}} \quad \text { and } \quad q_{[220]}=2 \sqrt{2} / a_{p, \mathrm{SL}} .
$$

These quantities are related to the measured diffraction angles $\omega$ and $\Theta$ by [17]:

$$
q_{[004]}=2[\sin \Theta \cos (\omega-\Theta)] / \lambda, \quad q_{[220]}=2[\sin \Theta \sin (\omega-\Theta)] / \lambda .
$$

The important advantage of the use of symmetric and asymmetric reciprocal space maps is the fact that $a_{n}$ and $a_{p}$ can be determined directly and as shown by Eq. (1) independently of each other and of any knowledge about the elastic constants.

\subsection{Short-period $S i_{m} G e_{n}$ superlattices}

All SL samples were grown by molecular beam epitaxy on (001) oriented non-miscut Si substrates. The nominal growth parameters for the buffer layers were the same in all three samples $A, B$, and $C$. A $100 \mathrm{~nm}$ thick Si layer was grown on top of the substrate followed by the step-graded buffer (B1), in which 
the Ge content was increased stepwise by $3 \%$ per $50 \mathrm{~nm}$ up to a total thickness of $650 \mathrm{~nm}$, i.e. to a nominal Ge content of $39 \%$. During the buffer growth, the temperature was decreased continuously from $600^{\circ} \mathrm{C}$ to $520^{\circ} \mathrm{C}$. Subsequently, a $550 \mathrm{~nm}$ thick $\mathrm{Si}_{0.6} \mathrm{Ge}_{0.4}$ alloy buffer layer (B2) was grown at $500^{\circ} \mathrm{C}$. Prior to the growth of the SL a monolayer of antimony was deposited as a surfactant. The SL $A$ sample (145 periods) was also grown at $T=500^{\circ} \mathrm{C}$. The SL samples $B$ and $C$ (100 periods each) were grown at $470^{\circ} \mathrm{C}$ and $450^{\circ} \mathrm{C}$, respectively. A $1 \mathrm{~nm}$ thick Si cap layer was deposited at the end of the growth.

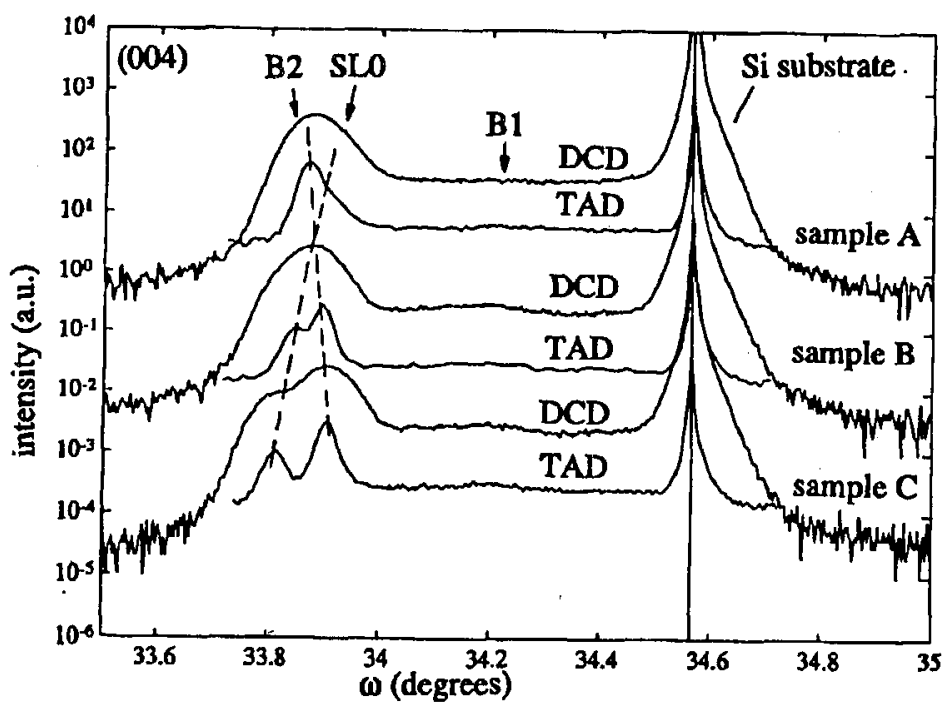

Fig. 4. $\omega / 2 \Theta$ rocking curves of the (004) Bragg reflection for three short-period $\mathrm{Si}_{9} \mathrm{Ge}_{6}$ SL's $(A, B$ and $C$ ) grown on thick step-graded SiGe alloy buffers (B1, $650 \mathrm{~nm}$ ) followed by a further $550 \mathrm{~nm}$ thick SiGe alloy buffer (B2). Data were taken with DCD optics (full lines). Diffracted intensity from the buffer B1 lies in between the substrate and SL0 peak, the $\mathrm{B} 2$ reflection overlaps in all DCD meaurements with the SL0 reflection. Using TAD optics, the SL0 and B2 reflections can be resolved as shown by the data plotted in this $\omega$ range, shifted downwards with respect to the DCD measurements for clarity. Traces for samples $B$ and $C$ have to be shifted upwards in intensity scale by 2 and 4 orders of magnitude, respectively.

Figure 4 shows DCD X-ray diffractograms along $q \|[004]$ for samples $A, B$, and $C$. The graded buffer B1 appears between the Si (004) substrate peak and the main superlattice peak SL0. It can be seen that the SL periods for the three samples are not identical, the samples $B$ and $C$ have a somewhat larger period than the sample $A$. Below the SL0 peak the buffer peak B2 is hidden for the sample $A$. Using TAD optics it is possible for the samples $B$ and $C$ to separate the SL0 from the buffer (B2) peaks due to the higher resolution as indicated in Fig. 4 by the three scans between the SL0 and substrate peaks, which are shifted downwards for clarity. The separation between buffer peak B2 and SL0 peak is shown in Fig. 5 


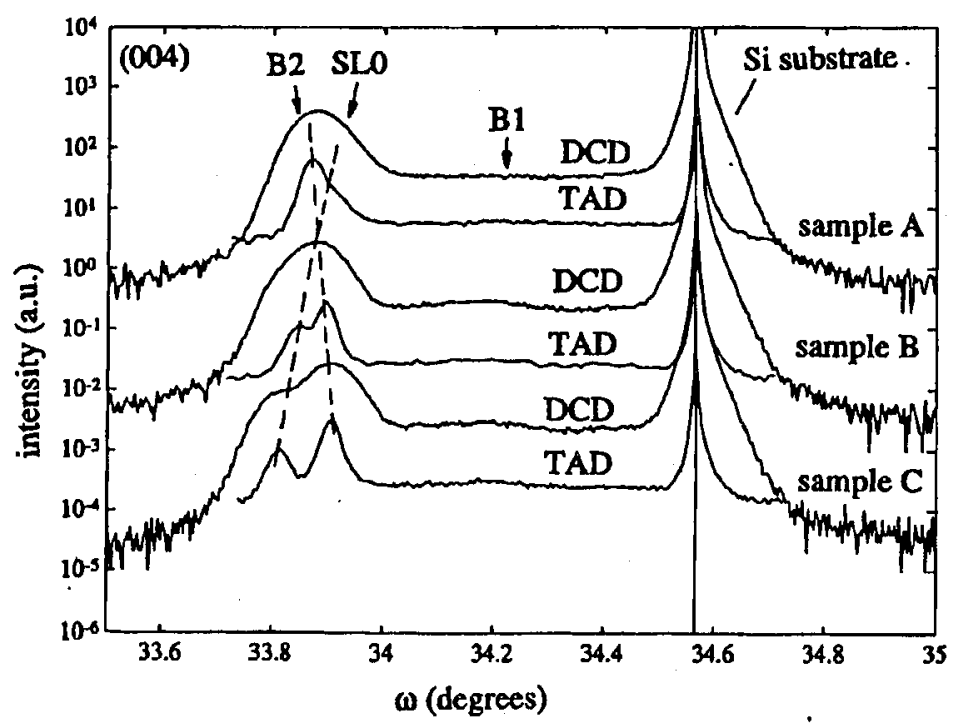

Fig. 5. Detail of Fig. 4 in the angular range of diffracted intensities from the Si substrate, the SLO and the from the buffers $B 2$ and B1, comparing results of DCD and TAD experiments. The dashed lines which indicate the peak positions of the SL0 and $\mathrm{B} 2$ reflections are just a guide for the eye. Intensity scales for SL samples $B$ and $C$ have to be shifted by 2 and 4 orders of magnitude, respectively.

for the three SL's comparing DCD with TAD scans. The peak positions of SL0 and buffer B2 depend both on the mean Ge content, as well as on their mean lattice constants in growth direction $a_{n, \mathrm{~B} 2}$ and $a_{n, \mathrm{SL}}$. The identification of the peaks SL0 and $\mathrm{B} 2$ relies on the position of the SL-1, SL-2 and SL+1 peaks (Fig. 4) and on the analysis of reciprocal lattice maps. From the rocking curves (Fig. 5) alone it is impossible to determine unambiguously whether for the buffer layer B2 in the three SL's the Ge content decreases from SL $A$ to SL $C$ or whether the degree of relaxation increases in the series SL $A$ to $C$. Similarly, it is difficult to judge whether in the SL's in the sequence $A$ to $C$ the mean Ge content decreases or whether SL $C$ is less free-standing with respect to the substrate than SL $A$.

However, in the following we demonstrate that reciprocal space maps describe much better the different relaxation status of buffer and superlattice in samples $A$ to $C$. The principle is shown schematically in Fig. 6, where regions of reciprocal space around the (004) and (224) RELP's for the Si substrate, the SiGe buffers and the zero-order SL peak SL0 are shown [21]. For clarity the satellite RELP's, accompanying SL0 along the [001] growth direction for both (004) and (224) RELP's, were not drawn in Fig. 6. For the initial stages of buffer growth, the in-plane lattice constant is identical with that of the Si substrate, whereas along growth direction the lattice constant is larger than that of the substrate and for pseudomorphic growth also larger than that of the relaxed SiGe alloy. The effect of partial strain relaxation on the position of the (224) buffer RELP is schematically 
[001] Growthdirection

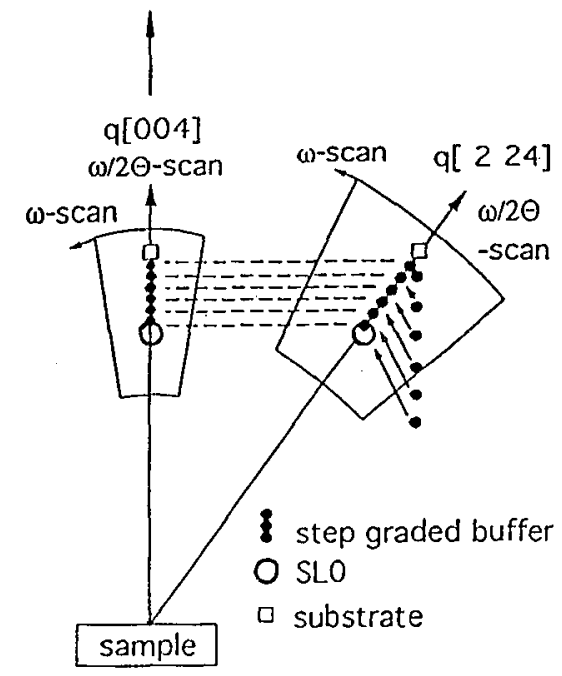

Fig. 6. Schematic diagram for (004) and (224) reciprocal lattice points of substrate $(\square)$, buffer $(\bullet)$ and zero-order SL peak (O) for SL's grown on thick, fully relaxed graded buffers. $\omega / 2 \Theta$ - and $\omega$-scan directions are depicted, as well as the areas in reciprocal space, which were scanned by a combination of both. The arrows indicate the path of the buffer RELP's $(\bullet)$ during relaxation.

indicated by arrows, the direction and length of which depend on the elastic constants and on the degree of relaxation. If the superlattice grows pseudomorphically on the buffer, the SL0 RELP lies along the [004] direction either above or below the corresponding buffer (224) RELP depending on the mean Ge contents of the buffer and the SL. For the thick step-graded buffer, the relaxation mechanism is sketched schematically in Fig. 6. If a complete relaxation of the individual slices, of which the buffer is composed, is achieved, then the corresponding RELP's finally lie along the [224] direction which connects (000) with the (224) substrate RELP. In such a case the superlattice with the proper mean composition (with respect to the top buffer layer) can grow virtually unaffected by the substrate. The in-plane lattice constants of the SL0 and the top buffer layer coincide when both the Ge content and the strain tensor components of the buffer are the same as the mean Ge content in the SL stack and the strain tensor components of the whole SL stack. In the case of a free-standing SL, within the SL layers the values of biaxial compression and dilation are unaffected by the substrate and depend just on the number of $\mathrm{Si}$ and $\mathrm{Ge}$ layers within one period.

In Fig. 7 reciprocal space maps around the (004) and (224) RELP's of sample $A$ are shown, which were derived from a series of $\omega / 2 \Theta$ scans with $\omega$-offsets using the proper transformation from angular space into reciprocal space. Contours of constant scattered intensity are shown using DCD optics in order to measure the 


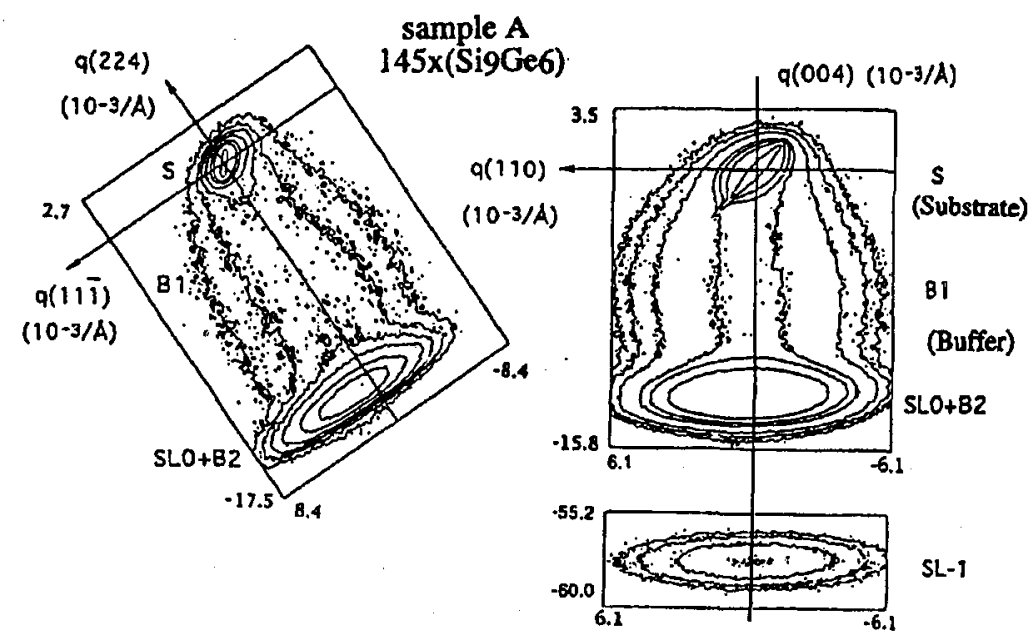

Fig. 7. Measured (224) and (004) reciprocal space maps for SL sample $A$ using DCD optics. The iso-intensity contours correspond to $0.5,1,2,10,20,2000 \mathrm{cps}$ in the (224) map and to $1,2,10,20,100,2000 \mathrm{cps}$ in the (004) map. The (224) buffer RELP lies below the center of the SL0 RELP along the [001] growth direction. The shift of the maximum intensity of the SL0 peak away from the $q \|$ [224] direction indicates a residual in plane strain.

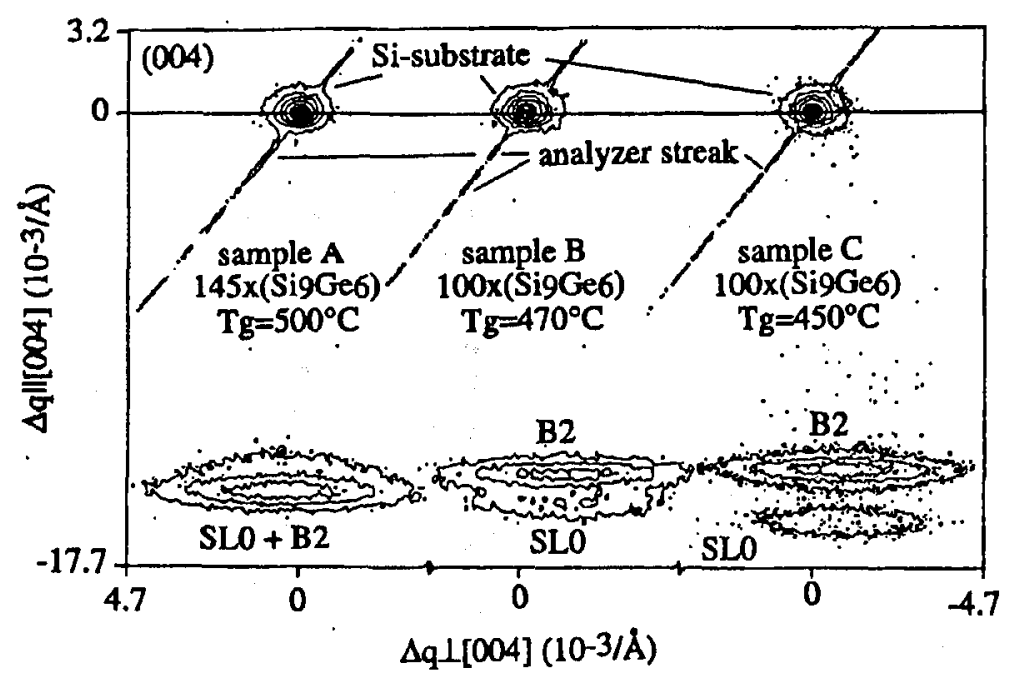

Fig. 8. Reciprocal space maps of (004) reflection measured with TAD optics for samples $A, B$ and $C$. Iso-intensity contours are shown in a logarithmic scale. Reflections from the buffers B1 are not visible because of their low intensities. The positions of SL0 and B2 peaks relative to the substrate indicate different strains within the SL stacks $A, B$ and $C$ and slightly different mean Ge content. 
low scattered intensity from the graded buffer region. The asymmetry around the substrate (004) RELP is an artifact (caused by the finite size of the reciprocal space probe, which is defined by the X-ray optics used) called "analyser streak" for TAD optics. From both RELP's around (004) and (224) it follows that all portions of the step-graded buffer (B1) are fully relaxed, because the intensity contours are almost symmetric around the $q \|$ [224] direction, which is not entirely the case for the SL0

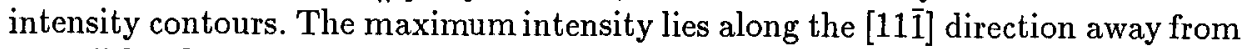
the $q \|[224]$ direction. The region of the SiGe alloy with constant Ge content (B2) yields intensity contours, which overlap in their positions with the SL0 contours. From the (004) reciprocal space map it follows that the tilt between SL, buffer, and substrate is negligible. From the reciprocal space maps, FWHM's of the intensity distribution parallel ([001]) and perpendicular ([110]) to the growth direction are deduced and for sample $A$ values of 365 and 1270 arcsec were found, respectively, for SL0, which remain unchanged for SL-1 within the accuracy of \pm 20 arcsec. From Fig. 7 it is obvious what potential is offered for immediate identification of the strain status of short-period SL's by reciprocal space maps. The symmetry of the intensity contours around the SL0 peaks indicates the absence of large scale strain gradients along the growth direction, and a rather statistical distribution of the mosaic blocks and a constant strain status within the SL stack [18].

In order to get precise strain data on the SL and the buffer B2, reciprocal space maps around the (004) (see Fig. 8) and (224) reflections were measured using TAD optics. As already mentioned the graded buffer regions do not appear in these maps because of their low intensity. Whereas in samples $A$ and $B$ the positions of SL0 and B2 RELP's overlap, they are clearly separated in sample $C$.

So far no numerical simulations of the intensity distribution around RELP's in reciprocal space were performed. From the reciprocal space coordinates of the intensity maxima of substrate, buffer layer and SLO RELP's the lattice constants parallel and normal to the growth direction are deduced (see Table).

In Figs. 9 and 10 we demonstrate the procedure for fitting $\omega-2 \Theta$ triple axis and DCD scans for the two SL's $B$ and $C$. For the fit we use all the strain values and the fixed SL periods given in Table. The remaining unknown parameter is the thickness ratio of the Si and Ge layers, which also shifts all SL peaks. We define a parameter $\beta$, which represents the deviation of the layer thicknesses (given in monolayers) from the nominal growth values $(m=9, n=6)$. Since the thickness of one monolayer of $\mathrm{Si}$ is slightly different from that of a monolayer of $\mathrm{Ge}$, an additional scaling factor $\alpha$ was included to retain the fixed value of the SL periods $P_{\mathrm{SL}}$ :

$$
P_{\mathrm{SL}}=\alpha\left[(m-\beta) M L_{\mathrm{Si}}+(n+\beta) M L_{\mathrm{Ge}}\right],
$$

where $M L_{\mathrm{Si}}$ and $M L_{\mathrm{Ge}}$ denote the monolayer thicknesses of the strained $\mathrm{Si}$ and $\mathrm{Ge}$ layers (measured in $\AA$ ). The lattice constants of the layers along growth direction are results of the strain analysis and are listed in Table. The results on the number of monolayers and their ratio, which deviates from $9 / 6=1.5$ are listed in Table as well. In Figs. 9 and 10, in the inserts secondary ion mass spectroscopy (SIMS) data on the Ge profile as a function of depth counted from the sample surface are shown. In the step-graded buffer $\mathrm{B} 1$ a slight deviation from the nominal 


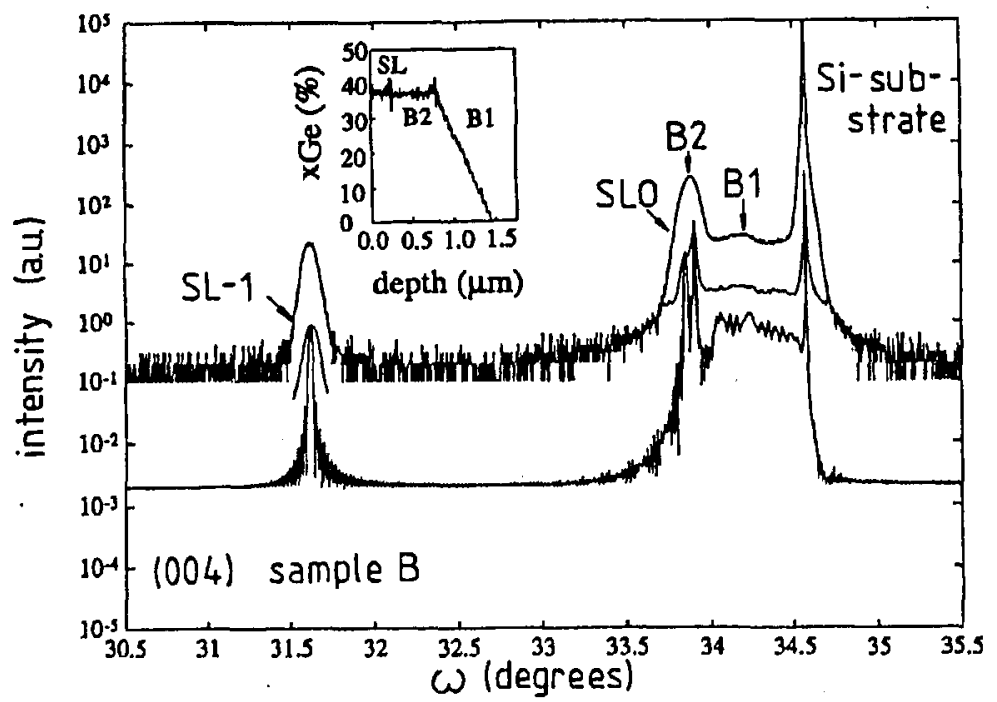

Fig. 9. $\omega / 2 \Theta$ scans of SL sample $B$ of the (004) Bragg reflection taken with DCD optics (top line) and TAD optics (center line, just for the $\omega$-range close to the SL0 and Si substrate reflections). Lowest line: results of a dynamical calculation of diffracted intensity using the parameters listed in Table. The insert shows the Ge content in the SL structure $B$ versus depth (from the surface), measured by SIMS.

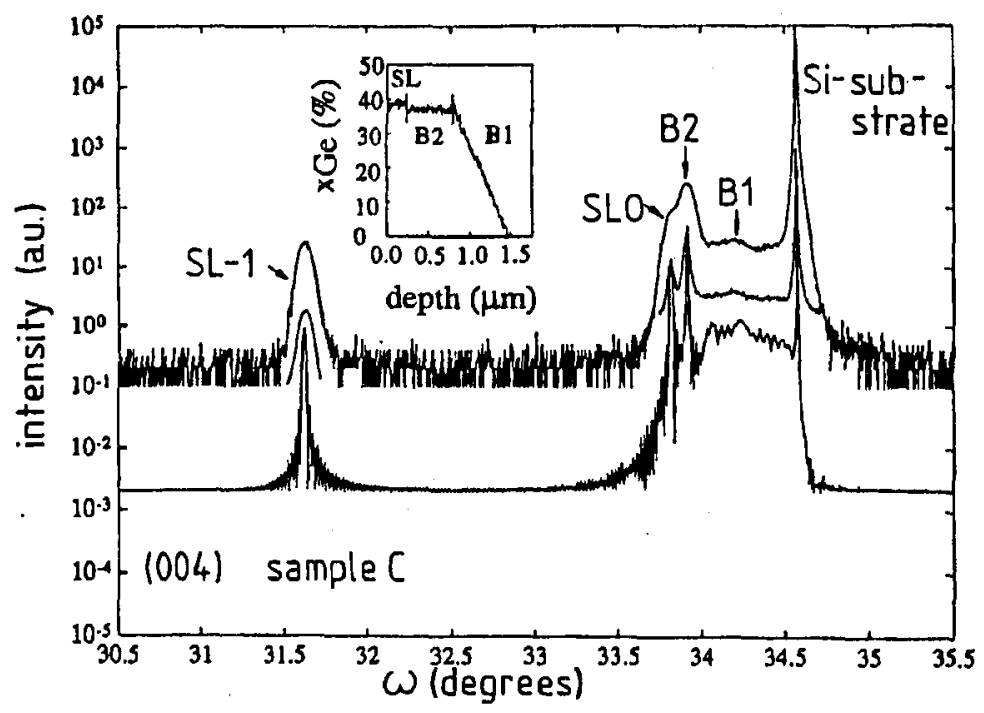

Fig. 10. As Fig. 9, but for SL sample $C$. 


\begin{tabular}{|c|c|c|c|c|c|c|}
\hline$\frac{13}{4}$ & 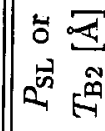 & 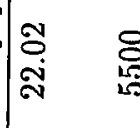 & 瓷 & $\begin{array}{c}8 \\
10 \\
10 \\
10\end{array}$ & S & $\begin{array}{l}8 \\
10 \\
10\end{array}$ \\
\hline & 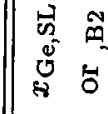 & $\mid$\begin{tabular}{l}
$\mathscr{\infty}$ \\
$\infty$ \\
\hdashline \\
0
\end{tabular} & 文 & $\stackrel{n}{n}$ & ঙ্ণে & $\stackrel{n}{\sigma}$ \\
\hline & 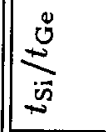 & 每 & 葛 & 1 & 告 & 1 \\
\hline & 总 & \begin{tabular}{ll}
$\infty$ & $N$ \\
\hdashline & $\infty$ \\
$\infty$ & 10
\end{tabular} & 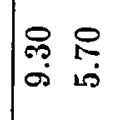 & 1 & 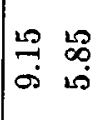 & I \\
\hline & है & 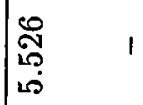 & $\begin{array}{l}\infty \\
\text { N } \\
10 \\
10 \\
10\end{array}$ & 1 & "ִ & 1 \\
\hline & $\underbrace{\sqrt[A]{2}}_{\omega} \underset{2}{2}$ & 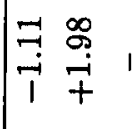 & 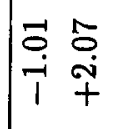 & 1 & 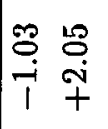 & 1 \\
\hline & ह5 & 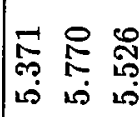 & 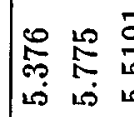 & $\begin{array}{l}\vec{\sigma} \\
1 \\
10 \\
10\end{array}$ & $\begin{array}{ll}10 \\
10 & 5 \\
00 & 5 \\
10 & 10\end{array}$ & $\begin{array}{l}O \\
\text { In } \\
10\end{array}$ \\
\hline 苟 & $\underbrace{\infty}_{\omega} \sqrt{2}$ & $\mid$\begin{tabular}{lcl}
\multirow{J}{*}{} & $\mathscr{0}$ & \\
$\vdots$ & $\stackrel{0}{*}$ & 1
\end{tabular} & 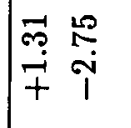 & 1 & 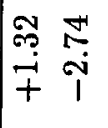 & I \\
\hline 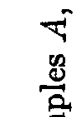 & 包要 & 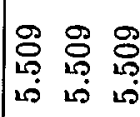 & 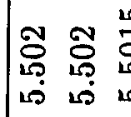 & 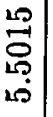 & 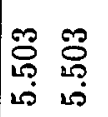 & 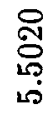 \\
\hline $\begin{array}{l}\text { 居 } \\
4 \\
\dot{0} \\
0\end{array}$ & 岕 & نี & 动 号 & ผै & i் & ติ \\
\hline छّ & 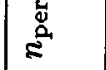 & $\stackrel{29}{-1}$ & 옹 & & 욤 & \\
\hline $\begin{array}{c}\tilde{a} \\
\tilde{0} \\
\tilde{\sigma}\end{array}$ & $\begin{array}{ll}60 & 0 \\
H & 0\end{array}$ & 욤 = & $\frac{R}{F}=$ & $\approx$ & 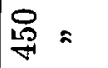 & 2 \\
\hline 壱 & 号 & $\square$ & $\infty$ & & 0 & \\
\hline
\end{tabular}


step grading of the Ge content was found which has been used in the dynamical simulation of the scattered X-ray intensities. The buffer B2 is somewhat thicker than the nominal $500 \mathrm{~nm}$ and has a Ge content $1-2 \%$ below the nominal value of $39 \%$. The mean Ge content in the SL, which results from the SIMS data is slightly below $40 \%$, in sample SL $C$ higher than in sample $B$ in agreement with the results of the X-ray strain analysis. The mean in-plane strain values of the SL's are $\varepsilon_{p, \mathrm{SL}}=-0.14 \%,-0.23 \%$ and $-0.27 \%$ for samples $A, B$, and $C$ respectively, which is a consequence of both the changes of the mean values of $a_{p}$ and of the thickness ratios.

\section{Conclusion}

In conclusion, double crystal and triple axis high resolution $\mathrm{X}$-ray diffraction measurements were used to characterize the strain status of a Si/SiGe MQW grown on $\mathrm{Si}(001)$ substrates. Furthermore, short-period $\mathrm{Si}_{m} \mathrm{Ge}_{n}$ SL's grown on step-graded SiGe alloy buffers were investigated. Two-dimensional reciprocal space maps around the (004) and (224) reciprocal lattice points reveal directly the strain status of the buffer layers, the MQW layers and the superlattice with respect to each other and to the Si substrate. For a proper choice of the Ge content, the thickness of the $\mathrm{Si}_{1-x} \mathrm{Ge}_{x}$ layers and their number, pseudomorphic growth is actually achieved. This results in extremely small FWIIM's of the iso-intensity contours around diffraction peaks, particularly for the direction perpendicular to the [001] growth direction (13 arcsec).

For the SL's, for a proper design of the graded SiGe alloy buffer, the $\mathrm{Si}_{9} \mathrm{Ge}_{6}$ SL's grow nearly free-standing, i.e. the topmost buffer layers offer the appropriate in-plane lattice constant for the SL's. This buffer design results in higher structural quality in comparison to SL's grown on thin $(200 \AA)$ alloy bufters with constant Ge content [20].

\section{Acknowledgment}

Work supported by FWF project No. 9119 and ESPRIT basic research aciion 7128 and Jubiläumsfonds der Österreichischen Nationalbank Projekt No. 4542. We thank A. Pesek for helpful discussions concerning the dynamical simulation of DCD data.

\section{References}

[1] E. Kasper, F. Schäfller, in: Semiconductors and Semimetals, Ed. T.P. Pearsall, Vol. 33, Academic Press, Boston 1991, p. 223.

[2] See e.g. a series of articles by G. Abstreiter, F. Schäfler, U. König, V.P. Kesan, K. Ismail, in Springer Series in Solid State Sciences, Vol. 101, Ed. G. Landwehr, Springer, Berlin 1992.

[3] R. People, Phys. Rev. B 32, 1405 (1985); M.M. Rieger, P. Vogl, Phys. Rev. B, in print.

[4] For a recent review see e.g.: W. Presting, A. Kibbel, M. Jaros, R.M. Turton, U. Menczigar, G. Abstreiter, H.G. Grimmeiss, Semicond. Sci. Technol. 7, 1127 (1992). 
[5] E.A. Fitzgerald, Y.-II. Xie, M.L. Green, D. Brasen, A.R. Kortan, J. Michel, Y.-J. Mii, B.E. Weir, Appl. Phys. Lett. 59, 811 (1991).

[6] F.K. LeGoues, B.S. Meyerson, J.F. Morar, Phys. Rev. Lett. 66, 2903 (1991).

[7] W. Jäger, D. Stenkamp, P. Ehrhardt, K. Leifer, W. Sybertz, H. Kibbel, H. Presting, E. Kasper, Thin Solid Films 222, 221 (1992).

[8] Y.J. Mii, Y.H. Xie, E.A. Fitzgerald, D. Monroe, F.A. Thiel, B.E. Weir, Appl. Phys. Lett. 59, 1611 (1991).

[9] F. Schäffler, D. Többen, II.J. Ilerzog, G. Abstreiter, B. Holländer, Semicond. Sci. Technol. 7, 260 (1992).

[10] J. Olajos, J. Engvall, II. Gimmeiss, U. Menczigar, G. Abstreiter, H. Kibbel, E. Kasper, H. Presting, Phys. Rev. B 46, 12857 (1992); U. Menczigar, G. Abstreiter, J. Olajos, H.G. Grimmeis, H. Kibbel, H. Presting, E. Kasper, Phys. Rev. $B$, in print.

[11] J. Engvall, J. Olajos, II.G. Grimmeis, H. Presting, H. Kibbel, E. Kasper, Appl. Phys. Lett., in print.

[12] D.C. Houghton, D.D. Perovic, J.M. Baribeau, G.C. Weatherly, J. Appl. Phys. 67, 1850 (1990).

[13] F.K. LeGoues, J.A. Ott, K. Eberl, S.S. Iyer, Appl. Phys. Lett. 61, 174 (1992).

[14] W. Koschinski, K. Dettmer, F.R. Kessler, J. Appl. Phys. 72, 471 (1992).

[15] P.J. Wang, M.S. Goorsky, B.S. Meyerson, F.K. LeGoues, Appl. Phys. Lett. 59, 814 (1991).

[16] P.F. Fewster, J. Appl. Crystallogr. 22, 64 (1989); 24, 178 (1991).

[17] E. Koppensteiner, T.W. Ryan, M. Ileuken, J. Söllner, J. Phys. D, Appl. Phys. 26, A35 (1993).

[18] V. Holy, J. Kubena, K. Ploog, Phys. Status Solidi B 162, 347 (1990).

[19] P.F. Fewster, Appl. Surf. Sci. 50, 9 (1991).

[20] T. Fromherz, E. Koppensteiner, M. IIelm, G. Bauer, J.F. Nützel, G. Abstreiter, Acta Phys. Pol. A 84, (1993).

[21] E. Koppensteiner, P. Hamberger, G. Bauer, A. Pesek, H. Kibbel, H. Presting, E. Kasper, Appl. Phys. Lett. 62, 1783 (1993). 